\title{
Optimal trajectories for efficient atomic transport without final excitation
}

\author{
Xi Chen, ${ }^{1,2}$ E. Torrontegui, ${ }^{1,3}$ Dionisis Stefanatos, ${ }^{4}$ Jr-Shin Li, ${ }^{4}$ and J. G. Muga ${ }^{1,3}$ \\ ${ }^{1}$ Departamento de Química-Física, UPV-EHU, Apartado 644, ES-48080 Bilbao, Spain \\ ${ }^{2}$ Department of Physics, Shanghai University, 200444 Shanghai, P.R. China \\ ${ }^{3}$ Max Planck Institute for the Physics of Complex Systems, Nöthnitzer Strasse 38, DE-01187 Dresden, Germany \\ ${ }^{4}$ Department of Electrical and System Engineering, Washington University, St. Louis, Missouri 63130, USA
}

(Received 5 August 2011; published 12 October 2011)

\begin{abstract}
We design optimal harmonic-trap trajectories to transport cold atoms without final excitation, combining an inverse engineering technique based on Lewis-Riesenfeld invariants with optimal control theory. Since actual traps are not really harmonic, we keep the relative displacement between the center of mass of the transport modes and the trap center bounded. Under this constraint, optimal protocols are found according to different physical criteria. The minimum time solution has a "bang-bang" form, and the minimum displacement solution is of "bang-off-bang" form. The optimal trajectories for minimizing the transient energy are also discussed.
\end{abstract}

DOI: 10.1103/PhysRevA.84.043415

PACS number(s): 37.10.Gh, 02.30.Yy, 03.65.Ca, 03.65.Nk

\section{INTRODUCTION}

Efficient transport of ultracold atoms and ions by moving the confining trap is an important goal in atomic physics [1-15], with applications to basic science, metrology, and quantum-information processing. A sufficiently slow, adiabatic motion is a simple way to transport the atoms without excitations or losses $[4,6,7]$. However, the long time required may become impractical, e.g., if a fast quantum-information operation is required [1,9], or counterproductive because of the accumulation of perturbations. Motivated by these drawbacks of adiabatic methods, several theoretical and experimental investigations have been devoted to making atomic transport fast and simultaneously faithful to the ideal final result of slow adiabatic transport [10-15].

These works on transport share concepts and techniques with other "shortcuts to adiabaticity" in expansion or compressions [12,16-24], rotations [25], and internal state population transfer and control [26-33]. Several approaches have been proposed, including counter-diabatic [26-28] or, equivalently, transitionless driving algorithms [29-31], optimal control theory [20], "fast-forward" scaling [12], and inverse engineering based on Lewis-Riesenfeld invariants [14-19,23,24,31-34].

We shall focus here on the invariant-based inverse engineering method, which relies on designing the Hamiltonian evolution so that the eigenvectors of corresponding invariants of motion become at initial and final times equal to the instantaneous eigenvectors of the Hamiltonian. This method provides in fact families of paths [31] which satisfy the initial and final boundary conditions, and thus guarantee the fast transitionless evolution, ideally in an arbitrarily short time. Given this freedom, it is natural to combine the invariant-based inverse method and optimal control theory to optimize the trajectory according to different physical criteria or operational constraints. For example, the time-dependent frequency of a harmonic trap expansion can be optimized with respect to time or to transient excitation energy, with a restriction of the allowed transient frequencies [35-37].

In this paper, we apply the invariant-based method complemented by optimal control theory to find optimal trajectories for fast atomic transport on harmonic traps without final vibrational excitation. Since actual traps are not really harmonic, we keep, as an imposed constraint, the relative displacement between the center of mass and the trap center bounded. We then optimize the trajectories according to different physical criteria: time minimization, (time-averaged) displacement minimization, and (time-averaged) transient energy minimization. The combination of the invariant-based method with optimal control and the use of the relative displacement as a constrained control variable differentiates the present article from our previous work [14], in which energy minimization for unconstrained displacement was applied on a heuristic basis.

\section{INVARIANT-BASED INVERSE ENGINEERING METHOD}

We consider here the harmonic transport of a particle of mass $m$ described by the time-dependent Hamiltonian

$$
H(t)=\frac{\hat{p}^{2}}{2 m}+\frac{1}{2} m \omega_{0}^{2}\left[\hat{q}-q_{0}(t)\right]^{2},
$$

where $\hat{q}$ and $\hat{p}$ are the position and momentum operators, $\omega_{0}$ is the constant (angular) frequency of the potential, and $q_{0}(t)$ is the position of the center of the harmonic trap. The corresponding quadratic-in-momentum Lewis-Riesenfeld invariant [34] has the form [38,39] (up to an arbitrary multiplicative constant)

$$
I(t)=\frac{1}{2 m}\left(\hat{p}-m \dot{q}_{c}\right)^{2}+\frac{1}{2} m \omega_{0}^{2}\left[\hat{q}-q_{c}(t)\right]^{2},
$$

where the dot represents a time derivative and the function $q_{c}(t)$ must satisfy the auxiliary equation

$$
\ddot{q}_{c}+\omega_{0}^{2}\left(q_{c}-q_{0}\right)=0,
$$

to guarantee the invariant condition

$$
\frac{d I(t)}{d t} \equiv \frac{\partial I(t)}{\partial t}+\frac{1}{i \hbar}[I(t), H(t)]=0 .
$$

Equation (3) is simply Newton's equation for a classical particle in the moving harmonic potential. 
An arbitrary solution of the time-dependent Schrödinger equation $i \hbar \partial_{t} \Psi(q, t)=H(t) \Psi(q, t)$, may be written in terms of "transport modes" $e^{i \alpha_{n}} \psi_{n}(q, t)$,

$$
\Psi(q, t)=\sum_{n} c_{n} e^{i \alpha_{n}} \psi_{n}(q, t),
$$

where $n=0,1, \ldots, c_{n}$ are time-independent coefficients, $\psi_{n}(q, t)$ are the orthonormal eigenvectors of the invariant $I(t)$ satisfying $I(t) \psi_{n}(q, t)=\lambda_{n} \psi_{n}(q, t)$, with real timeindependent $\lambda_{n}$, and the Lewis-Riesenfeld phase is defined as

$$
\alpha_{n}(t)=\frac{1}{\hbar} \int_{0}^{t}\left\langle\psi_{n}\left(t^{\prime}\right)\left|i \hbar \frac{\partial}{\partial t^{\prime}}-H\left(t^{\prime}\right)\right| \psi_{n}\left(t^{\prime}\right)\right\rangle d t^{\prime} .
$$

For the harmonic trap considered here [39],

$$
\begin{aligned}
\psi_{n}(q, t)= & \frac{1}{\left(2^{n} n !\right)^{1 / 2}}\left(\frac{m \omega_{0}}{\pi \hbar}\right)^{1 / 4} \exp \left[-\frac{m \omega_{0}}{2 \hbar}\left(q-q_{c}\right)^{2}\right] \\
& \times \exp \left(i \frac{m \dot{q}_{c} q}{\hbar}\right) H_{n}\left[\left(\frac{m \omega_{0}}{\hbar}\right)^{1 / 2}\left(q-q_{c}\right)\right]
\end{aligned}
$$

(the $H_{n}$ are Hermite polynomials), i.e., $q_{c}$ is the center of mass of the transport modes. ${ }^{1}$ Substituting Eq. (7) into Eq. (6),

$$
\alpha_{n}=-\frac{1}{\hbar} \int_{0}^{t} d t^{\prime}\left(\lambda_{n}+\frac{m \dot{q}_{c}^{2}}{2}\right),
$$

where $\lambda_{n}=E_{n}=(n+1 / 2) \hbar \omega_{0}$. The instantaneous average energy for a transport mode can be obtained from Eqs. (1) and (7),

$$
\left\langle\psi_{n}(t)|H(t)| \psi_{n}(t)\right\rangle=\hbar \omega_{0}(n+1 / 2)+E_{c}+E_{p},
$$

where the first, "internal" contribution remains constant for each $n, E_{c}=m \dot{q}_{c}^{2} / 2$, and $E_{p}=\frac{1}{2} m \omega_{0}^{2}\left(q_{c}-q_{0}\right)^{2}$ has the form of a potential energy for a classical particle. The instantaneous average potential energy can be written as

$$
\langle V(t)\rangle=\frac{\hbar \omega_{0}}{2}(n+1 / 2)+E_{p} .
$$

Suppose that the harmonic trap is displaced from $q_{0}(0)=0$ to $q_{0}\left(t_{f}\right)=d(d>0)$ in a time $t_{f}$. The trajectory $q_{0}(t)$ of the trap can be inverse engineered by designing first an appropriate classical trajectory $q_{c}(t)$. To avoid vibrational excitation at the final time we impose the conditions

$$
\begin{array}{ccc}
q_{c}(0)=0, & \dot{q}_{c}(0)=0, & \ddot{q}_{c}(0)=0, \\
q_{c}\left(t_{f}\right)=d, & \dot{q}_{c}\left(t_{f}\right)=0, & \ddot{q}_{c}\left(t_{f}\right)=0,
\end{array}
$$

which, along with Eq. (3), imply also that

$$
q_{0}(0)=0, \quad q_{0}\left(t_{f}\right)=d .
$$

The above boundary conditions guarantee the commutativity of $I(t)$ and $H(t)$ at $t=0$ and $t=t_{f}$; that is, the transport modes coincide with the eigenvectors of the instantaneous Hamiltonian at $t=0$ and $t=t_{f} . q_{c}(t)$ can be interpolated by a simple polynomial ansatz that satisfies these boundary

\footnotetext{
${ }^{1}$ We shall also refer to $q_{c}$ as the "classical trajectory." Note that the center of mass of a linear combination of transport modes may be displaced with respect to $q_{c}$.
}

conditions. Once $q_{c}(t)$ is fixed, we get the trap trajectory $q_{0}(t)$ from Eq. (3).

As discussed later in more detail, the boundary conditions on the second derivatives and consequently the conditions for $q_{0}$ in Eq. (13) are special, in the sense that we shall allow for discontinuities in the acceleration $\ddot{q}_{c}$ at the edge times (in fact also elsewhere). Physically this means that the trap is ideally allowed to be displaced suddenly a finite distance, whereas the velocity $\dot{q}_{c}$ and the trajectory $q_{c}$ remain always continuous.

Viewed as a mathematical problem, there is no lower bound for $t_{f}$ [14]. However, there are always some physical limits in the laboratory related, for instance, to spatial or energy constraints, leading to a finite $t_{f}$.

\section{OPTIMAL CONTROL PROBLEM WITH CONSTRAINED RELATIVE DISPLACEMENT}

We begin with the equation of motion, Eq. (3), for the classical particle in the harmonic trap, and set, for compactness and to follow the usual conventions in optimal control theory, a new notation,

$$
x_{1}=q_{c}, \quad x_{2}=\dot{q}_{c}, \quad u(t)=q_{c}-q_{0},
$$

where $x_{1}, x_{2}$ are the components of a "state vector" $\mathbf{x}$, and the relative displacement between the trap and the classical trajectory $u(t)$ is considered as the (scalar) control function. The physical motivation behind this control is that actual traps are not really harmonic, so the relative displacement should be kept bounded to avoid anharmonic perturbations. Equation (3) becomes

$$
\begin{gathered}
\dot{x}_{1}=x_{2}, \\
\dot{x}_{2}=-\omega_{0}^{2} u .
\end{gathered}
$$

The optimal-control problem is to find $|u(t)| \leqslant \delta$ for some fixed bound $\delta$, with $u(0)=0$ and $u\left(t_{f}\right)=0$ such that the system starts at $\left\{x_{1}(0)=0, x_{2}(0)=0\right\}$, ends up at $\left\{x_{1}\left(t_{f}\right)=\right.$ $\left.d, x_{2}\left(t_{f}\right)=0\right\}$, and minimizes a cost function $J$.

The boundary conditions for $x_{1}$ and $x_{2}$ can be equivalently considered as those for $q_{c}$ and $\dot{q}_{c}$. The boundary conditions for $u(t)$ are equivalent to those for $q_{0}$ and, through Eq. (3), equivalent to those for $\ddot{q}_{c}$, so there are totally six boundary conditions, as in Eqs. (11) and (12). A natural way to understand the boundary conditions on $u(t)$ is to consider that $u(t)=0$ for $t \leqslant 0$ and $t \geqslant t_{f}$, so the classical trajectory and the trap center coincide before and after the transport. We will consider cost functions that are not affected by the isolated values $u(0)$ and $u\left(t_{f}\right)$, minimizing the transport time, the displacement, or the energy, and solve the control problem in the interval $\left(0, t_{f}\right)$. In order to match the boundary conditions at the initial and final times, the optimal control obtained may be complemented by appropriate jumps at these points which do not affect the cost. We use Pontryagin's maximum principle, which provides necessary conditions for optimality [40]. Generally, to minimize the cost function

$$
J(u)=\int_{0}^{t_{f}} g(\mathbf{x}(t), u) d t,
$$

the maximum principle states that for the dynamical system $\dot{\mathbf{x}}=\mathbf{f}(\mathbf{x}(t), u)$, the coordinates of the extremal vector $\mathbf{x}(t)$ and of the corresponding adjoint state $\mathbf{p}(t)$ formed by Lagrange 
multipliers, $p_{1}, p_{2}$, fulfill Hamilton's equations for a control Hamiltonian $H_{c}$,

$$
\begin{gathered}
\dot{\mathbf{x}}=\frac{\partial H_{c}}{\partial \mathbf{p}}, \\
\dot{\mathbf{p}}=-\frac{\partial H_{c}}{\partial \mathbf{x}},
\end{gathered}
$$

where $H_{c}$ is defined as

$$
H_{c}(\mathbf{p}(t), \mathbf{x}(t), u)=p_{0} g(\mathbf{x}(t), u)+\mathbf{p}^{T} \cdot \mathbf{f}(\mathbf{x}(t), u) .
$$

The superscript $T$ used here denotes the transpose of a vector, and $p_{0}<0$ can be chosen for convenience since it amounts to multiplying the cost function by a constant. The (augmented) vector with components $\left(p_{0}, p_{1}, p_{2}\right)$ is nonzero and continuous. For almost all $0 \leqslant t \leqslant t_{f}$, the function $H_{c}(\mathbf{p}(t), \mathbf{x}(t), u)$ attains its maximum at $u=u(t)$, and $H_{c}(\mathbf{p}(t), \mathbf{x}(t), u(t))=c$, where $c$ is constant.

\section{A. Time minimization}

We discuss now the time-minimization optimal-control problem with a constrained relative displacement, that is, $|u(t)|=\left|q_{c}-q_{0}\right| \leqslant \delta$, which means $E_{p} \leqslant \frac{1}{2} m \omega_{0}^{2} \delta^{2}$. To find the minimal time $t_{f}$ we define the cost function

$$
J_{T}=\int_{0}^{t_{f}} d t=t_{f} .
$$

The control Hamiltonian $H_{c}(\mathbf{p}(t), \mathbf{x}(t), u)$ is

$$
H_{c}\left(p_{1}, p_{2}, x_{1}, x_{2}, u\right)=p_{0}+p_{1} x_{2}-p_{2} \omega_{0}^{2} u .
$$

With the control Hamiltonian, Eq. (19) gives the following costate equations:

$$
\begin{gathered}
\dot{p}_{1}=0, \\
\dot{p}_{2}=-p_{1} .
\end{gathered}
$$

They are solved easily as $p_{1}=c_{1}$ and $p_{2}=-c_{1} t+c_{2}$ with constants $c_{1}$ and $c_{2}$. According to the Pontryagin's maximum principle, the time-optimal control $u(t)$ maximizes the control Hamiltonian in Eq. (22).

Since the control Hamiltonian is a linear function of the control function $u(t)$, the optimal control that maximizes $H_{c}$ is determined by the sign of $p_{2}$, when $u(t)$ is bounded, $|u(t)| \leqslant \delta$. When $p_{2} \neq 0$, the optimal control in the duration $t_{f}$ is given by

$$
u(t)= \begin{cases}-\delta, & p_{2}>0, \\ \delta, & p_{2}<0 .\end{cases}
$$

If $p_{2}=0$ for some time interval, then $p_{1}=0$ from Eq. (24), and $p_{0}=0$ from Eq. (22), since $H_{c}=0$ for the time-optimal problem [40], in contrast with the maximum principle that requires $\left(p_{0}, p_{1}, p_{2}\right) \neq 0$. Thus $p_{2}$ can be zero only at isolated points, the switching times. The solutions of the costate functions in Eqs. (23) and (24) imply that the function of $p_{2}$ depends linearly on time $t$, so that the sign of $p_{2}$ cannot change more than once. Since the final point is $\left(x_{1}, x_{2}\right)=(d, 0), d>0$,
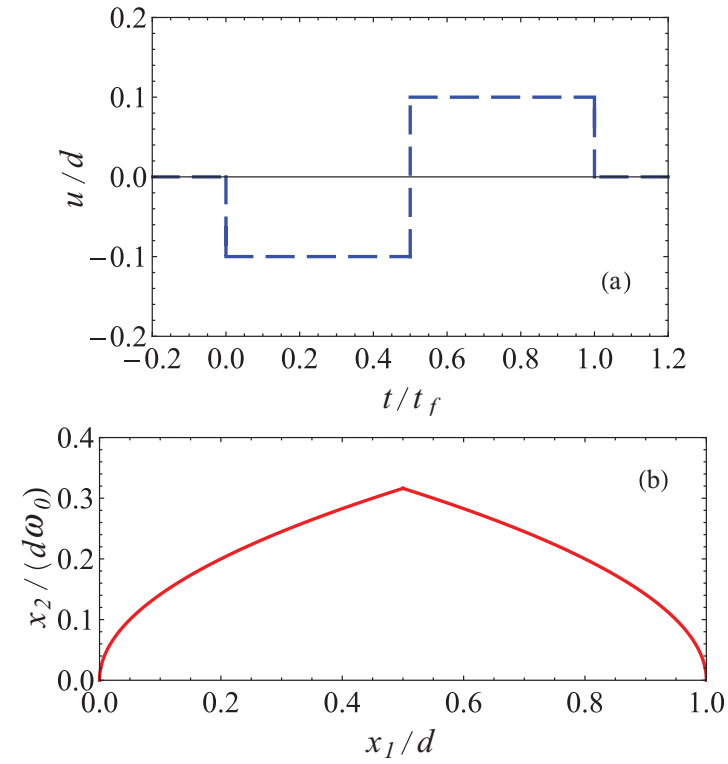

FIG. 1. (Color online) (a) The control function (dashed blue line) for time-optimal problem, and (b) the corresponding trajectory (solid red line) for $\omega_{0}=2 \pi \times 50 \mathrm{~Hz}, \delta=0.1 d$, and $t_{f}=20 \mathrm{~ms}$ given by Eq. (28).

the appropriate control sequence is of "bang-bang" (piecewise constant) type,

$$
u(t)=\left\{\begin{array}{lc}
0, & t \leqslant 0, \\
-\delta, & 0<t<t_{1}, \\
\delta, & t_{1}<t<t_{f}, \\
0, & t \geqslant t_{f},
\end{array}\right.
$$

with only one intermediate switching time at $t_{1}$, as shown in Fig. 1(a). The saturation of the control is typical of time minimization problems.

Substituting $u(t)$ into the classical Eq. (3), and using the boundary conditions in Eqs. (11) and (12), we find the optimal classical trajectory

$$
q_{c}(t)=\left\{\begin{array}{lc}
0, & t \leqslant 0, \\
\omega_{0}^{2} \delta t^{2} / 2, & 0<t<t_{1}, \\
d-\omega_{0}^{2} \delta\left(t-t_{f}\right)^{2} / 2, & t_{1}<t<t_{f}, \\
d, & t \geqslant t_{f},
\end{array}\right.
$$

and the corresponding trajectory for the harmonic trap is

$$
q_{0}(t)=\left\{\begin{array}{lc}
0, & t \leqslant 0, \\
\left(1+\omega_{0}^{2} t^{2} / 2\right) \delta, & 0<t<t_{1}, \\
d-\left[\omega_{0}^{2}\left(t-t_{f}\right)^{2} / 2+1\right] \delta, & t_{1}<t<t_{f}, \\
d, & t \geqslant t_{f} .
\end{array}\right.
$$

Figure 1(b) illustrates the time-optimal trajectory with one switching time. Solving the system of Eqs. (15) and (16), one can find the switching time $t_{1}$ and final time $t_{f}$,

$$
\begin{gathered}
t_{1}=\frac{t_{f}}{2}, \\
t_{f}=\frac{2}{\omega_{0}} \sqrt{\frac{d}{\delta}},
\end{gathered}
$$




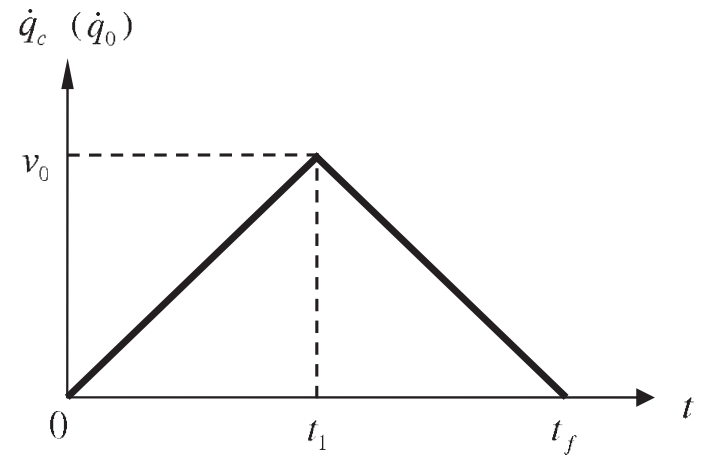

FIG. 2. Velocity profiles of harmonic trap and particles for time minimization, where the switching time $t_{1}=t_{f} / 2$.

by imposing continuity on $x_{1}$ and $x_{2}$. For the "bang-bang" control, the motion of the trap has discontinuities, while we impose continuity for the trajectory of the particle. As illustrated by Fig. 2, the velocities of particle and trap become equal,

$$
\dot{q}_{c}=\dot{q}_{0}= \begin{cases}\omega_{0}^{2} \delta t, & 0<t<t_{1}, \\ -\omega_{0}^{2} \delta\left(t-t_{f}\right), & t_{1}<t<t_{f},\end{cases}
$$

since $u(t)$ is piecewise constant during the bang-bang control. The maximum velocity occurs at $t=t_{f} / 2$,

$$
v_{0}=\omega_{0}^{2} \delta t_{f} / 2=\omega_{0} \sqrt{d \delta}
$$

which is restricted by the imposed bound $|u(t)| \leqslant \delta$. In addition, the instantaneous potential energy $\langle V\rangle$ is constant, and

$$
E_{p}=\frac{1}{2} m \omega_{0}^{2} \delta^{2}=\frac{8 m d^{2}}{\omega_{0}^{2} t_{f}^{4}} .
$$

If we loosen the bound by increasing $\delta$, the maximum velocity and the instantaneous potential energy increase, and the final time may be shortened.

\section{B. Displacement minimization}

In this section, we minimize the integral, or time-average of the relative displacement, which is equivalent to a minimal control-effort problem. To this end, the cost function can be defined as

$$
J_{D}=\int_{0}^{t_{f}}|u(t)| d t=\int_{0}^{t_{f}}\left|q_{c}-q_{0}\right| d t,
$$

and the control Hamiltonian is

$$
H_{c}\left(p_{1}, p_{2}, x_{1}, x_{2}, u\right)=p_{0}|u|+p_{1} x_{2}-p_{2} \omega_{0}^{2} u,
$$

which leads to the same costate equations for $p_{1}$ and $p_{2}$ in Eqs. (23) and (24). Thus, we obtain $p_{1}=c_{1}$ and $p_{2}=-c_{1} t+$ $c_{2}$ with constants $c_{1}$ and $c_{2}$ from the costate equations. We use for convenience the normalization $p_{0}=-\omega_{0}^{2}$. Disregarding $u$-independent terms in $H_{c}$, the function of $u(t)$ that we have to maximize is

$$
-\omega_{0}^{2}\left(|u|+p_{2} u\right)= \begin{cases}-\omega_{0}^{2}\left(1+p_{2}\right) u, & u \geqslant 0, \\ \omega_{0}^{2}\left(1-p_{2}\right) u, & u \leqslant 0 .\end{cases}
$$

According to Pontryagin's maximum principle, when $u(t)$ is bounded, $|u(t)| \leqslant \delta$, the control function is

$$
u(t)=\left\{\begin{array}{lc}
-\delta, & p_{2}>1, \\
0, & -1<p_{2}<1, \\
\delta, & p_{2}<-1,
\end{array}\right.
$$

which maximizes the control Hamiltonian in Eq. (33). Switchings of the optimal control occur at isolated instants when $p_{2}= \pm 1$ and $\dot{p}_{2} \neq 0$. In principle we may also consider

$$
p_{2}= \begin{cases}-1, & u \geqslant 0 \\ 1, & u \leqslant 0\end{cases}
$$

in the whole interval from 0 to $t_{f}$. This may occur if $c_{1}=0$ and $c_{2}= \pm 1$, and thus $p_{1}=0$. The optimal-control problem becomes a singular-control case [37,41-43], in which the maximization of the function in Eq. (34) with respect to $u$ gives us no useful information. However, when $p_{2}=-1$ and $u \geqslant 0$, the initial point $(0,0)$ is driven to $\left(x_{1}, x_{2}\right)$ with $x_{1}, x_{2} \leqslant 0$, according to Eqs. (15) and (16), so the target point $(d, 0)$ cannot be reached. Similarly, for $p_{2}=1$ and $u \leqslant 0,(d, 0)$ at $t_{f}$ cannot be reached either because $u$ should be positive for some time interval. Thus, the singular control plays no role in this problem and the optimal control is given by Eq. (35).

Notice that whereas in the minimum-time problem discussed above, the optimal control is bang-bang, the minimaldisplacement control can be described as "bang-off-bang." Owing to the properties of costate equations, the bang-off-bang trajectory with two switching times $t_{1}$ and $t_{1}+t_{2}$ can be
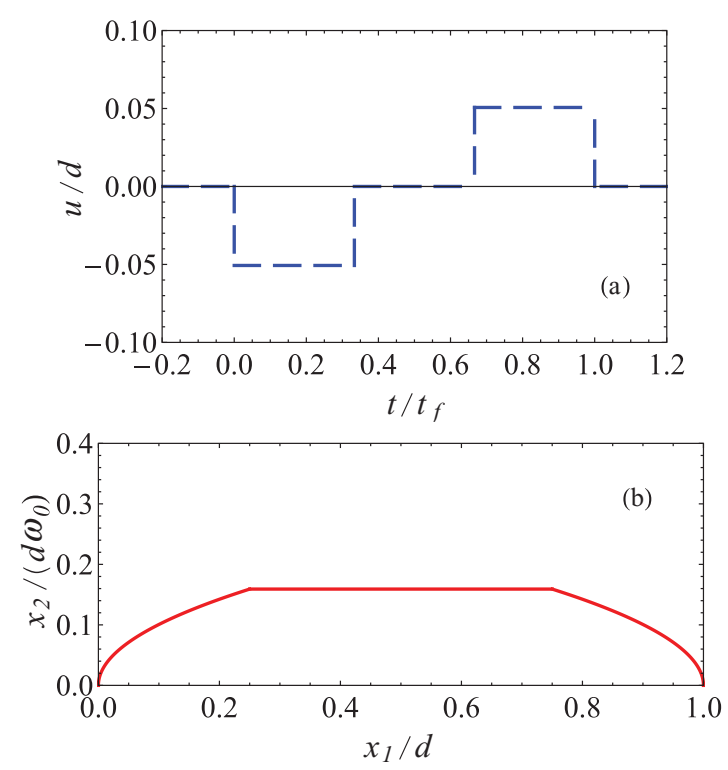

FIG. 3. (Color online) (a) Control function (dashed blue line) for the displacement-optimal problem and (b) corresponding trajectory (solid red line) for $t_{f}=30 \mathrm{~ms}, \delta=9 d / 2 \omega_{0}^{2} t_{f}^{2}$, and $\omega_{0}=2 \pi \times 50 \mathrm{~Hz}$. 
described by, see Fig. 3(a),

$$
u(t)=\left\{\begin{array}{lc}
0, & t \leqslant 0, \\
-\delta, & 0<t<t_{1}, \\
0, & t_{1}<t<t_{1}+t_{2}, \\
\delta, & t_{1}+t_{2}<t<t_{f}, \\
0, & t \geqslant t_{f} .
\end{array}\right.
$$

Substituting the control function $u(t)$ into Eq. (3), using the boundary conditions in Eqs. (11) and (12), and imposing the continuity of $q_{c}$ at the two switching times, the optimal classical trajectory, as shown in Fig. 3(b), is finally given by

$$
q_{c}(t)=\left\{\begin{array}{lc}
0, & t \leqslant 0, \\
\omega_{0}^{2} \delta t^{2} / 2, & 0<t<t_{1}, \\
v_{0} t-v_{0}^{2} /\left(2 \omega_{0}^{2} \delta\right), & t_{1}<t<t_{1}+t_{2}, \\
d-\omega_{0}^{2} \delta\left(t-t_{f}\right)^{2} / 2, & t_{1}+t_{2}<t<t_{f}, \\
d, & t \geqslant t_{f},
\end{array}\right.
$$

which results in the following optimal trap trajectory,

$$
q_{0}(t)=\left\{\begin{array}{lc}
0, & t \leqslant 0, \\
\left(1+\omega_{0}^{2} t^{2} / 2\right) \delta, & 0<t<t_{1}, \\
v_{0} t-v_{0}^{2} /\left(2 \omega_{0}^{2} \delta\right), & t_{1}<t<t_{1}+t_{2}, \\
d-\left[\omega_{0}^{2}\left(t-t_{f}\right)^{2} / 2+1\right] \delta, & t_{1}+t_{2}<t<t_{f}, \\
d, & t \geqslant t_{f} .
\end{array}\right.
$$

As in the bang-bang time minimization, the velocities of particle and trap are equal, see Fig. 4,

$$
\dot{q}_{c}=\dot{q}_{0}=\left\{\begin{array}{lc}
\omega_{0}^{2} \delta t, & 0<t<t_{1}, \\
v_{0}, & t_{1}<t<t_{1}+t_{2}, \\
-\omega_{0}^{2} \delta\left(t-t_{f}\right), & t_{1}+t_{2}<t<t_{f},
\end{array}\right.
$$

where $v_{0}$ is the maximum velocity of trap motion in the trajectory, which will be determined later. With the boundary conditions for $x_{1}$ and $x_{2}$ at $t=t_{1}$ and $t=t_{1}+t_{2}$, the switching times can be calculated as

$$
\begin{gathered}
t_{1}=\frac{v_{0}}{\omega_{0}^{2} \delta}, \\
t_{2}=\frac{d}{v_{0}}-\frac{v_{0}}{\omega_{0}^{2} \delta} .
\end{gathered}
$$

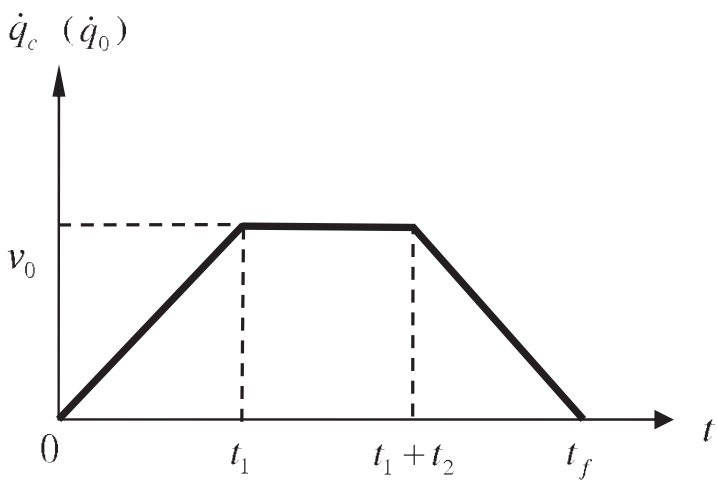

FIG. 4. Velocity profiles of harmonic trap and particles for displacement minimization with two switching times $t_{1}$ and $t_{1}+t_{2}$.
As a consequence, the final time is

$$
t_{f}=2 t_{1}+t_{2}=\frac{d}{v_{0}}+\frac{v_{0}}{\omega_{0}^{2} \delta} \geqslant \frac{2}{\omega_{0}} \sqrt{\frac{d}{\delta}} .
$$

Since the final time $t_{f}$ is fixed, there are three possible cases: (i) When $t_{f}>\left(2 / \omega_{0}\right) \sqrt{d / \delta}$, the maximal velocity $v_{0}$ can be solved from Eq. (43) as

$$
v_{0}^{ \pm}=\frac{\omega_{0}^{2} \delta t_{f}}{2}\left(1 \pm \sqrt{1-\frac{4 d}{\omega_{0}^{2} t_{f}^{2} \delta}}\right),
$$

where $v_{0}^{+}$should be ignored, because it leads to $2 t_{1}>t_{f}$. (ii) If $t_{f}=\left(2 / \omega_{0}\right) \sqrt{d / \delta}$, the maximum velocity is $v_{0}=$ $\omega_{0} \sqrt{d \delta}$, thus $t_{1}=t_{f} / 2$ and $t_{2}=0$. The trajectory in this case is reduced to that of the time-optimal control problem. (iii) When the time $t_{f}$ is less than $\left(2 / \omega_{0}\right) \sqrt{d / \delta}$, there is no real solution to $v_{0}$ and no solution to displacement minimization.

Interestingly, the bang-off-bang trajectory for displacement minimization may be related to the trajectory used for atomic transport in Ref. [3], where the shift velocity $\dot{q}_{0}$ was increased linearly during a quarter of the spatial transported distance $d / 4$, then kept constant for $d / 2$, and during the last quarter finally ramped back to zero. The corresponding (continuous) trap trajectory $q_{0}$ is exactly given by the function in Eq. (38) with

$$
\delta=\frac{9 d}{2 \omega_{0}^{2} t_{f}^{2}},
$$
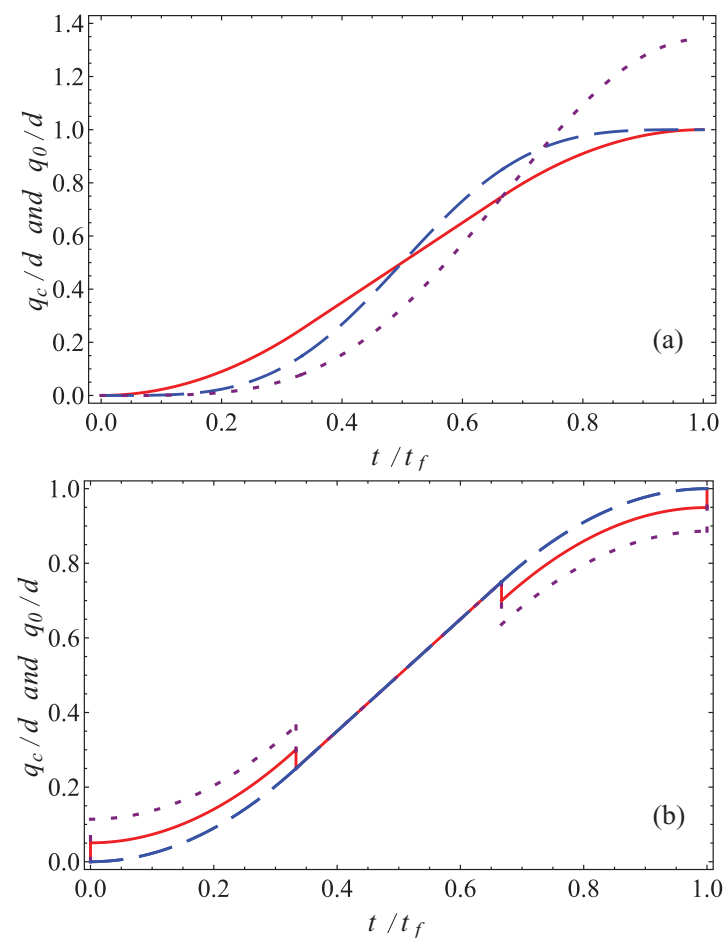

FIG. 5. (Color online) (a) Comparison of trajectories $q_{c}$ (dashed blue line: $t_{f}=30 \mathrm{~ms}$ and dotted purple line: $t_{f}=20 \mathrm{~ms}$ ) and $q_{0}$ (solid red line) for the protocol in Ref. [3]. Note that $t_{f}=3 \pi / \omega_{0}=30$ $\mathrm{ms}$ is a magic time without final excitation [14]. (b) Comparison of trajectories $q_{c}$ (dashed blue line) and $q_{0}$ (solid red line: $t_{f}=20 \mathrm{~ms}$ and dotted purple line: $t_{f}=30 \mathrm{~ms}$ ) in the displacement-optimal control. $\omega_{0}=2 \pi \times 50 \mathrm{~Hz}$ and $\delta=9 d / 2 \omega_{0}^{2} t_{f}^{2}$. 
maximal velocity

$$
v_{0}=\frac{3 d}{2 t_{f}},
$$

and switching times

$$
t_{1}=\frac{1}{3} t_{f}=\frac{d}{2 v_{0}}, \quad t_{2}=\frac{1}{3} t_{f}=\frac{d}{2 v_{0}} .
$$

An example is depicted in Fig. 5(a). The associated $q_{c}$ results from solving Eq. (3) for this trap motion and in general will not satisfy the boundary conditions at $t_{f}$, Eq. (12), except for a discrete set of "magic times" [14]. Figure 5(a) shows $q_{c}$ for two different values of $t_{f}$, one of them being a magic time.

Contrast this to the result of the optimization when choosing the maximum displacement $\delta$ as in Eq. (45). According to Eq. (44), $v_{0}$ is also given by Eq. (46), and, from Eqs. (41) and (42), the switching times are as in Eq. (47), so the optimal classical trajectory $q_{c}$ would exactly coincide with the continuous $q_{0}$ set in the experiments [3], avoiding final excitations for any $t_{f}$. Nevertheless the trap trajectory $q_{0}$ necessary to implement that displacement-optimal classical trajectory, according to Eq. (39), includes finite jumps by $\pm \delta$ at $t=0, t_{1}, t_{1}+t_{2}$, and $t_{f}$, as depicted in Fig. 5(b).

Returning now to the general case, the time-averaged potential energy for the optimal trajectory is

$$
\overline{E_{p}}=\frac{\int_{0}^{t_{f}} E_{p} d t}{t_{f}}=\frac{m \omega_{0}^{2} \delta^{2} t_{1}}{t_{f}},
$$

where $t_{1}=v_{0} / \omega_{0}^{2} \delta$ is given by

$$
t_{1}=\frac{t_{f}}{2}\left(1-\sqrt{1-\frac{4 d}{\omega_{0}^{2} t_{f}^{2} \delta}}\right) .
$$

As a result,

$$
\overline{E_{p}}=\frac{1}{2} m \omega_{0}^{2} \delta^{2}\left(1-\sqrt{1-\frac{4 d}{\omega_{0}^{2} t_{f}^{2} \delta}}\right) .
$$

For example, when $\delta=9 d / 2 \omega_{0}^{2} t_{f}^{2}$ and $t_{1}=t_{f} / 3$ are chosen as discussed above, the time-averaged potential energy is $\overline{E_{p}}=27 m d^{2} / 4 \omega_{0}^{2} t_{f}^{4}$, which is less than the (constant) potential energy $E_{p}=8 m d^{2} / \omega_{0}^{2} t_{f}^{4}$ for the time-optimal control problem.

\section{Energy minimization}

The instantaneous potential energy $\langle V(t)\rangle$ is given in Eq. (10). To minimize the potential energy average for a given mode $n$ and fixed transport time $t_{f}$, the cost function can be defined as

$$
J_{E}=\int_{0}^{t_{f}} E_{p} d t=\int_{0}^{t_{f}} \frac{1}{2} m \omega_{0}^{2} u^{2} d t,
$$

and the control Hamiltonian is

$$
H_{c}=-p_{0} \frac{1}{2} m \omega_{0}^{2} u^{2}+p_{1} x_{2}-p_{2} \omega_{0}^{2} u,
$$

which gives two costate equations, Eqs. (23) and (24). The solutions are as before $p_{1}=c_{1}$ and $p_{2}=-c_{1} t+c_{2}$, with constants $c_{1}$ and $c_{2}$. For the normalization $p_{0}=-1 / m$ the function of $u(t)$ that we have to maximize is $-u^{2} / 2-p_{2} u$.
Here we start with the case of "unbounded control," i.e., without imposing any constraints on the displacement, and we will show how this is related to the physically interesting case where the control is bounded. To maximize $-u^{2} / 2-p_{2} u$, the control function is found to be

$$
u(t)=-p_{2},
$$

and the classical Eq. (3), $\ddot{q}_{c}=-\omega_{0}^{2} u$, gives the optimal trajectory

$$
q_{c}=-\frac{1}{6} c_{1} \omega_{0}^{2} t^{3}+\frac{1}{2} c_{2} \omega_{0}^{2} t^{2}+c_{3} t+c_{4} .
$$

Using the boundary conditions for $q_{c}$ and $\dot{q}_{c}$ in Eqs. (11) and (12), we find $c_{1}=12 d / \omega_{0}^{2} t_{f}^{3}, c_{2}=6 d / \omega_{0}^{2} t_{f}^{2}, c_{3}=0$, and $c_{4}=$ 0 . Clearly, Eq. (54) does not satisfy the boundary conditions for $\ddot{q}_{c}$ in Eqs. (11) and (12). To guarantee $u(t)=0$ at $t \leqslant 0$ and $t \geqslant t_{f}$ and match the boundary conditions, the control function $u(t)$ has to be complemented by the appropriate jumps at these two edges. Consequently, the control function for unbounded control, see Fig. 6(a), is found to be

$$
u(t)=\left\{\begin{array}{lc}
0, & t \leqslant 0, \\
\frac{6 d}{\omega_{0}^{2} t_{f}^{2}}\left(2 \frac{t}{t_{f}}-1\right), & 0<t<t_{f}, \\
0, & t \geqslant t_{f} .
\end{array}\right.
$$

As shown in Fig. 6(b), the optimal classical trajectory for unbounded control finally becomes

$$
q_{c}=\left\{\begin{array}{lc}
0, & t \leqslant 0, \\
\frac{d t^{2}}{t_{f}^{2}}\left(3-2 \frac{t}{t_{f}}\right), & 0<t<t_{f}, \\
d, & t \geqslant t_{f},
\end{array}\right.
$$

where the trajectory $q_{c}$ in the interval $\left(0, t_{f}\right)$ is in agreement with the result obtained in Ref. [14] using the Euler-Lagrange
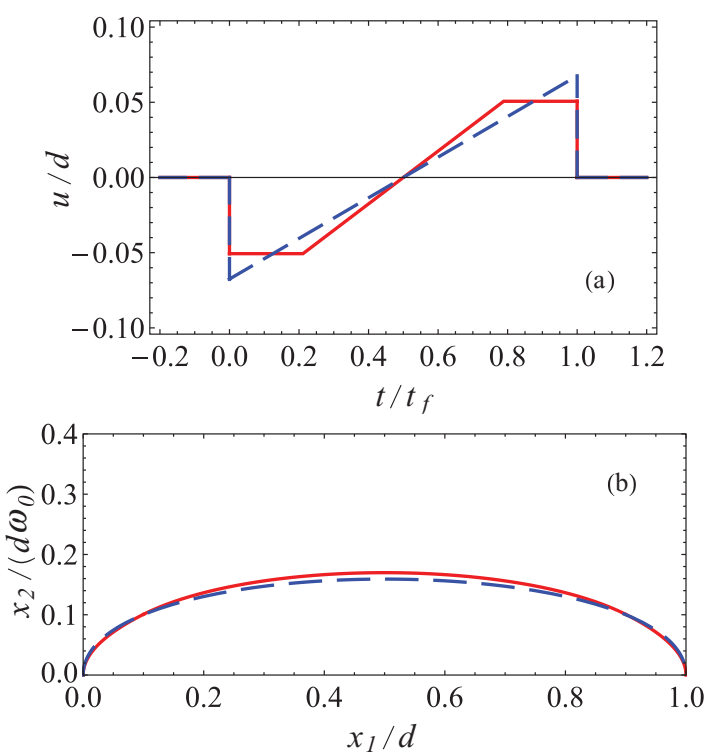

FIG. 6. (Color online) (a) Control functions for the energyoptimal problem with bounded (solid red line) and unbounded (dashed blue line) controls, and (b) corresponding trajectories in the cases of bounded (solid red line) and unbounded (dashed blue line) controls. The parameters are the same as in Fig. 3. 
equation. In this case, the time-averaged minimal potential energy is

$$
{\overline{E_{p}}}^{\min }=\frac{\int_{0}^{t_{f}} E_{p} d t}{t_{f}}=\frac{6 m d^{2}}{\omega_{0}^{2} t_{f}^{4}},
$$

which gives a lower bound for the time-averaged potential energy of any other trajectories satisfying all the boundary conditions, $\overline{E_{p}} \geqslant 6 m d^{2} / \omega_{0}^{2} t_{f}^{4}$. Note that, in spite of not having preimposed a bound for the displacement, the optimal trajectory obeys $|u(t)| \leqslant \delta_{0}=6 d / \omega_{0}^{2} t_{f}^{2}$. For the bounded control, i.e., when $|u(t)| \leqslant \delta$ is imposed, if $\delta \geqslant \delta_{0}$ the unbounded solution is the optimal one. [The value of $\delta_{0}$ can be obtained in the bounded control case by requiring $t_{1} \geqslant 0$, see Eq. (67) below.]

When the bound, $|u(t)| \leqslant \delta$, is imposed, the control function is

$$
u(t)=\left\{\begin{array}{lc}
-\delta, & p_{2}>\delta, \\
-p_{2}, & -\delta<p_{2}<\delta, \\
\delta, & p_{2}<-\delta,
\end{array}\right.
$$

to achieve the maximum value of the control Hamiltonian $H_{c}$. As before, the linear $p_{2}$ implies two switching times $t_{1}$ and $t_{1}+t_{2}$. To make the control function continuous at $t_{1}$ and $t_{1}+t_{2}$, it has the form shown in Fig. 6(a),

$$
u(t)=\left\{\begin{array}{lc}
0, & t \leqslant 0, \\
-\delta, & 0<t<t_{1}, \\
c_{1}\left(t-t_{f} / 2\right), & t_{1}<t<t_{1}+t_{2}, \\
\delta, & t_{1}+t_{2}<t<t_{f}, \\
0, & t \geqslant t_{f},
\end{array}\right.
$$

where, because of $t_{f}=2 t_{1}+t_{2}$ due to the symmetry, the two switching times $t_{1}$ and $t_{2}$ are given by

$$
t_{2}=2 \delta / c_{1}, \quad t_{1}=\frac{t_{f}-2 \delta / c_{1}}{2} .
$$

Unlike the time-minimization and displacementminimization problems, the control function here is not piecewise constant, so the velocities of the classical particle and the trap are not equal during the second segment from $t_{1}$ to $t_{1}+t_{2}$, see Fig. 7. According to the control function in

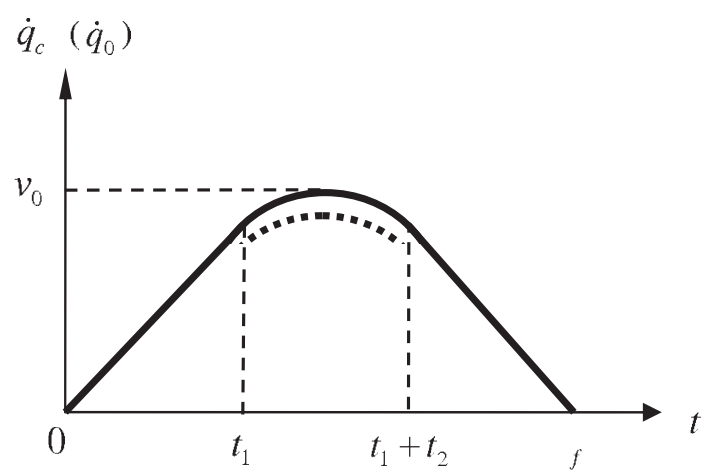

FIG. 7. Velocity profiles of harmonic trap (dashed line) and classical trajectory (solid line) for energy minimization with two switching times $t_{1}$ and $t_{1}+t_{2}$.
Eq. (59), imposing the boundary conditions for $x_{2}$ at $t=0$ and $t=t_{f}$, the velocity for the classical trajectory is

$$
\dot{q}_{c}=\left\{\begin{array}{lc}
\omega_{0}^{2} \delta t, & 0<t<t_{1}, \\
-\frac{1}{2} \omega_{0}^{2} c_{1}\left(t-\frac{t_{f}}{2}\right)^{2}+v_{0}, & t_{1}<t<t_{1}+t_{2}, \\
-\omega_{0}^{2} \delta\left(t-t_{f}\right), & t_{1}+t_{2}<t<t_{f} .
\end{array}\right.
$$

and $\dot{q}_{0}=\dot{q}_{c}-\dot{u}$ gives the velocity profile of the trap,

$$
\dot{q}_{0}=\left\{\begin{array}{lc}
\omega_{0}^{2} \delta t, & 0<t<t_{1}, \\
-\frac{1}{2} \omega_{0}^{2} c_{1}\left(t-\frac{t_{f}}{2}\right)^{2}+v_{0}-c_{1}, & t_{1}<t<t_{1}+t_{2}, \\
-\omega_{0}^{2} \delta\left(t-t_{f}\right), & t_{1}+t_{2}<t<t_{f},
\end{array}\right.
$$

where $v_{0}$ is the maximum velocity. With $t_{2}=2 \delta / c_{1}$, and further imposing continuity of $x_{2}$ at $t=t_{1}$ and $t=t_{1}+t_{2}$, we find

$$
t_{1}=\frac{v_{0}}{\omega_{0}^{2} \delta}-\frac{\delta}{2 c_{1}},
$$

which finally leads to $t_{f}=2 t_{1}+t_{2}$,

$$
t_{f}=\frac{2 v_{0}}{\omega_{0}^{2} \delta}+\frac{\delta}{c_{1}} .
$$

Solving Eqs. (63) and (64), the parameters $c_{1}$ and $v_{0}$ are given by

$$
c_{1}=\frac{2 \delta}{t_{f}-2 t_{1}}, \quad v_{0}=\frac{1}{4} \omega_{0}^{2} \delta\left(t_{f}+2 t_{1}\right) .
$$

Thus, $c_{2}=\delta t_{f} /\left(t_{f}-2 t_{1}\right)$. So far, $c_{1}, c_{2}$, and $v_{0}$ are all functions of $t_{1}$. To determine $t_{1}$ we write the optimal-energy classical trajectory from Eq. (61),

$q_{c}(t)=\left\{\begin{array}{lc}0, & t \leqslant 0, \\ \frac{1}{2} \omega_{0}^{2} t^{2} \delta, & 0<t<t_{1}, \\ -\frac{1}{6} \omega_{0}^{2} c_{1}\left(t-\frac{t_{f}}{2}\right)^{3}+v_{0} t+c_{3}, & t_{1}<t<t_{1}+t_{2}, \\ d-\frac{1}{2} \omega_{0}^{2}\left(t-t_{f}\right)^{2} \delta, & t_{1}+t_{2}<t<t_{f}, \\ d, & t \geqslant t_{f} .\end{array}\right.$

By imposing its continuity at $t=t_{1}$ and $t=t_{1}+t_{2}, c_{3}$ and $t_{1}$ can be solved as

$$
\begin{gathered}
c_{3}=\frac{1}{2}\left(d-v_{0} t_{f}\right), \\
t_{1}=\frac{t_{f}}{2}\left(1-\sqrt{3} \sqrt{1-\frac{4 d}{\omega_{0}^{2} t_{f}^{2} \delta}}\right),
\end{gathered}
$$

where the other unphysical solution should be neglected. Once $t_{1}$ is fixed, $c_{j}(j=1,2,3)$ are available, and $v_{0}$ is given by

$$
v_{0}=\frac{\omega_{0}^{2} \delta t_{f}}{2}\left(1-\frac{\sqrt{3}}{2} \sqrt{1-\frac{4 d}{\omega_{0}^{2} t_{f}^{2} \delta}}\right),
$$

which is smaller than the maximum velocity for the displacement-optimal trajectory. A trajectory with minimal energy and bounded control is depicted in Fig. 6(b). It is seen from Eqs. (67) and (68) that for a real $t_{1}$ and $v_{0}$, $t_{f} \geqslant\left(2 / \omega_{0}\right) \sqrt{d / \delta}$ should be satisfied. In the particular case $t_{f}=\left(2 / \omega_{0}\right) \sqrt{d / \delta}$, the maximum velocity is $v_{0}=\omega_{0} \sqrt{d \delta}$, thus $t_{1}=t_{f} / 2$ and $t_{2}=0$. Like for displacement minimization, the 


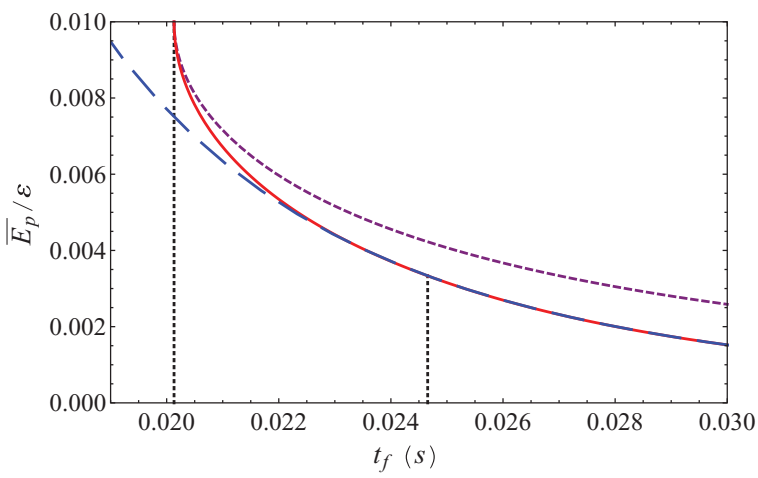

FIG. 8. (Color online) Dependence of time-averaged energies $\overline{E_{p}}$ on $t_{f}$ with $\delta=0.1 d$, for energy minimization with bounded control (red solid line), unbounded control, Eq. (57) (blue dashed line), and displacement minimization (purple dotted line). Parameters are $\omega_{0}=$ $2 \pi \times 50 \mathrm{~Hz}, \varepsilon=m \omega_{0}^{2} d^{2} / 2$, and mass of rubidium 87 . Vertical lines delimit the interval $4 d /\left(\delta \omega_{0}^{2}\right) \leqslant t_{f}^{2} \leqslant 6 d /\left(\delta \omega_{0}^{2}\right)$.

trajectory in this case is reduced again to that of the timeoptimal control problem. Moreover, to make $t_{1}$ non-negative, $t_{f}$ should be less than $\left(\sqrt{6} / \omega_{0}\right) \sqrt{d / \delta}$. If $t_{f}>\left(\sqrt{6} / \omega_{0}\right) \sqrt{d / \delta}$, the optimal trajectory is the one in the unbounded-control case, as commented before. In other words, $\delta>\delta_{0}=6 d / \omega_{0}^{2} t_{f}^{2}$. As a result, the five-segment form in Eq. (59) applies for the interval $4 d /\left(\delta \omega_{0}^{2}\right) \leqslant t_{f}^{2} \leqslant 6 d /\left(\delta \omega_{0}^{2}\right)$ delimited by vertical lines in Fig. 8. There is no solution for smaller times, whereas the solution becomes the one for unbounded control for larger times.

In this energy-optimal trajectory, the time-averaged potential energy $\overline{E_{p}}$ should be minimized. The cost function in Eq. (51) becomes

$$
J_{E}=m \omega_{0}^{2} \delta^{2} t_{1}+\frac{1}{6} m \omega_{0}^{2} \delta^{2} t_{2}
$$

and therefore

$$
\overline{E_{p}}=\frac{\int_{0}^{t_{f}} E_{p} d t}{t_{f}}=m \omega_{0}^{2} \delta^{2}\left(\frac{2 t_{1}}{3 t_{f}}+\frac{1}{6}\right),
$$

which finally results in

$$
\overline{E_{p}}=\frac{1}{2} m \omega_{0}^{2} \delta^{2}\left(1-\frac{2 \sqrt{3}}{3} \sqrt{1-\frac{4 d}{\omega_{0}^{2} t_{f}^{2} \delta}}\right) .
$$

In Fig. 8, we compare this to the (larger) average energy for the displacement-optimal problem, Eq. (50), and the lower bound Eq. (57), and also demonstrate that the lower energy bound can be realized when $t_{f}>\left(\sqrt{6} / \omega_{0}\right) \sqrt{d / \delta}$.

\section{DISCUSSION AND CONCLUSIONS}

We have proposed optimal protocols for fast atomic transport in harmonic traps combining the invariant-based inverse engineering method and optimal control theory. Optimal trajectories with bang-bang and bang-off-bang forms are, respectively, obtained for time minimization and displacement minimization with constrained displacement between the trap center and the center of mass of the particle density in the transport modes. The transient energies for bounded and unbounded displacement are also minimized. As an example of the potentiality of these optimization methods, let us apply the adiabaticity criterion $t_{f}^{2} \gg m d^{2} /\left(2 \hbar \omega_{0}\right)$ to harmonic transport with constant velocity of an atom of rubidium 87 a distance $d=1 \mathrm{~mm}$, and trap frequency $\omega_{0} /(2 \pi)=50 \mathrm{~Hz}$. This gives $t_{f} \gg 1.4 \mathrm{~s}$. Suppose that the transport is done with a Gaussian dipole trap [44] with waist $w_{0}=50 \mu \mathrm{m}$ and wavelength $\lambda=850 \mathrm{~nm}$. The corresponding Rayleigh range is $z_{R}=\pi w_{0}^{2} / \lambda=0.92 \mathrm{~cm}$. Setting a displacement bound $\delta=$ $z_{R} / 100$ to stay in the harmonic regime gives a minimal time, Eq. (28), of $21 \mathrm{~ms}$, a significant reduction with respect to the adiabatic result. There are many different conditions regarding times, distances, constraints, and the transported system for which the proposed trap trajectories or their smoothed versions could be implemented using magnetic or optical traps driven by several mechanisms [14]. In this respect the numerical values of this example or the ones used for the figures are only for illustration. A much broader range of parameters may be considered, ranging from microseconds to seconds, or from micrometers to meters. Specific implementations would of course benefit from a more detailed analysis taking into account their peculiarities and technical limitations regarding trap features and stability, trap speed limits, coherence times, switching times, or geometric constraints.

In the time-optimal problem, the minimal time, Eq. (28), corresponds to a fixed constraint: $\delta$ is the maximum displacement allowed. Consistent with this, no solutions are found for displacement- and energy-minimization problems for transport times shorter than the minimal time, i.e., for $t_{f}<$ $\left(2 / \omega_{0}\right) \sqrt{d / \delta}$. To achieve fast and faithful transport in shorter times, an "energy price" must be paid by increasing $\delta$, which, in real traps, will also produce errors because of anharmonicities. The relation between the minimal (time-averaged) energy and the transport time $t_{f}$ obtained here is not at all trivial, in particular they are not simply inversely proportional, see, e.g., Eq. (57) or (71), as one might naively expect from the form of time-energy uncertainty relations. The scaling laws found are also peculiar to transport. For example the minimal energy in Eq. (57) depends on $t_{f}^{-4}$ instead of the $t_{f}^{-2}$ dependence applicable to engineered trap expansions [18].

In a previous work on invariants and transport [14], the energy bound for $\overline{E_{p}}$ was found using the Euler-Lagrange equation. Here we have shown how to realize this bound by allowing the discontinuous acceleration of the trap at $t=0$ and $t=t_{f}$ in the unbounded control optimization, and also finite jumps in the trap position. In principle these and other discontinuities found in the trap motion design could be avoided by imposing appropriate bounds (for example, on the rate of change of the control) and using a powerful pseudospectral numerical optimization method $[35,45,46]$ to address the corresponding more complex optimal-control problem. One may also approximate the jumps with fast switching technology. Accelerations which are effectively discontinuous compared to atomic or trap motion time scales are currently implemented in transport experiments $[5,10]$, and effectively sudden trap displacements could be based on rapid phase switches of one of the lasers forming a standing wave by electro-optical modulators. Alternatively, one can switch off and on very rapidly a beam shifting the focus, in less than $100 \mathrm{~ns}$. Further work is required to analyze the perturbations induced by these operations as well as the deviation from the optimal results and bounds (in time or energy) due to continuous solutions to the trap motion. 
While the strategy in this work has been to keep the atom displacement small to avoid anharmonic effects, anharmonicity could be dealt with in a completely different way using the protocols for anharmonic transport described in Ref. [14], which require a compensation of inertial forces in the frame of the trap. This may be feasible or not depending on the accelerations imparted, and the corresponding optimization is also left for future work. Last but not least, the present results may be extended to Bose-Einstein condensates following [15], and Tonks-Girardeau gases could be treated as well with a simple generalization [17].

\section{ACKNOWLEDGMENTS}

We thank D. Guéry-Odelin, G. C. Hegerfeldt, D. Meschede, and A. Ruschhaupt for useful discussions. We acknowledge funding by the Basque Government (Grant No. IT472-10) and Ministerio de Ciencia e Innovación (FIS2009-12773C02-01). X. C. thanks the Juan de la Cierva Programme, the National Natural Science Foundation of China (Grant Nos. 60806041 and 61176118) and the Shanghai Leading Academic Discipline Program (Grant No. S30105); E.T., the Basque Government (Grant No. BFI08.151); and J.-S. Li, the AFOSR Grant FA9550-10-1-0146.
[1] M. A. Rowe et al., Quantum Inf. Comput. 4, 257 (2002).

[2] R. Reichle, D. Leibfried, R. B. Blakestad, J. Britton, J. D. Jost, E. Knill, C. Langer, R. Ozeri, S. Seidelin, and D. J. Wineland, Fortschr. Phys. 54, 666 (2006).

[3] W. Hänsel, J. Reichel, P. Hommelhoff, and T. W. Hänsch, Phys. Rev. Lett. 86, 608 (2001).

[4] W. Hänsel, P. Hommelhoff, T. W. Hänsch, and J. Reichel, Nature 413, 498 (2001).

[5] D. Schrader, S. Kuhr, W. Alt, M. Müller, V. Gomer, and D. Meschede, Appl. Phys. B 73, 819 (2001).

[6] T. L. Gustavson, A. P. Chikkatur, A. E. Leanhardt, A. Görlitz, S. Gupta, D. E. Pritchard, and W. Ketterle, Phys. Rev. Lett. 88, 020401 (2001).

[7] S. Schmid, G. Thalhammer, K. Winkler, F. Lang, and J. H. Denschlag, New J. Phys. 8, 159 (2006).

[8] T. Lahaye, G. Reinaudi, Z. Wang, A. Couvert, and D. GuéryOdelin, Phys. Rev. A 74, 033622 (2006).

[9] H.-K. Lau and D. F. V. James, Phys. Rev. A 83, 062330 (2011).

[10] A. Couvert, T. Kawalec, G. Reinaudi, and D. Guéry-Odelin, Europhys. Lett. 83, 13001 (2008).

[11] M. Murphy, L. Jiang, N. Khaneja, and T. Calarco, Phys. Rev. A 79, 020301(R) (2009).

[12] S. Masuda and K. Nakamura, Proc. R. Soc. A 466, 1135 (2010).

[13] D. Chen, H. Zhang, X. Xu, T. Li, and Y. Wang, Appl. Phys. Lett. 96, 134103 (2010).

[14] E. Torrontegui, S. Ibáñez, X. Chen, A. Ruschhaupt, D. GuéryOdelin, and J. G. Muga, Phys. Rev. A 83, 013415 (2011), and references therein.

[15] E. Torrontegui, X. Chen, M. Modugno, S. Schmidt, A. Ruschhaupt, and J. G. Muga, e-print arXiv:1103.2532.

[16] J. G. Muga, X. Chen, A. Ruschhaupt, and D. Guéry-Odelin, J. Phys. B 42, 241001 (2009).

[17] X. Chen, A. Ruschhaupt, S. Schmidt, A. del Campo, D. Guéry-Odelin, and J. G. Muga, Phys. Rev. Lett. 104, 063002 (2010).

[18] X. Chen and J. G. Muga, Phys. Rev. A 82, 053403 (2010).

[19] J. G. Muga, X. Chen, S. S. Ibáñez, I. Lizuain, and A. Ruschhaupt, J. Phys. B 43, 085509 (2010).

[20] P. Salamon, K. H. Hoffmann, Y. Rezek, and R. Kosloff, PhysChemChemPhys 11, 1027 (2009).

[21] Y. Li, L.-A. Wu, and Z.-D. Wang, Phys. Rev. A 83, 043804 (2011).

[22] A. del Campo, Phys. Rev. A 84, 031606(R) (2011).
[23] J. F. Schaff, X.-L. Song, P. Vignolo, and G. Labeyrie, Phys. Rev. A 82, 033430 (2010).

[24] J. F. Schaff, X. L. Song, P. Capuzzi, P. Vignolo, and G. Labeyrie, Europhys. Lett. 93, 23001 (2011).

[25] J. F. Schaff, P. Capuzzi, G. Labeyrie, and P. Vignolo, e-print arXiv:1105.2119v1.

[26] M. Demirplak and S. A. Rice, J. Phys. Chem. A 107, 9937 (2003).

[27] M. Demirplak and S. A. Rice, J. Phys. Chem. B 109, 6838 (2005).

[28] M. Demirplak and S. A. Rice, J. Chem. Phys. 129, 154111 (2008).

[29] M. V. Berry, J. Phys. A 42, 365303 (2009).

[30] X. Chen, I. Lizuain, A. Ruschhaupt, D. Guéry-Odelin, and J. G. Muga, Phys. Rev. Lett. 105, 123003 (2010).

[31] X. Chen, E. Torrontegui, and J. G. Muga, Phys. Rev. A 83, 062116 (2011)

[32] S. Ibáñez, S. Martínez-Garaot, X. Chen, E. Torrontegui, and J. G. Muga, Phys. Rev. A 84, 023415 (2011).

[33] M. S. Sarandy, E. I. Duzzioni, and R. M. Serra, Phys. Lett. A 375, 3343 (2011).

[34] H. R. Lewis and W. B. Riesenfeld, J. Math. Phys. 10, 1458 (1969).

[35] D. Stefanatos, J. Ruths, and J.-S. Li, Phys. Rev. A 82, 063422 (2010).

[36] D. Stefanatos and J.-S. Li, e-print arXiv:1012.3646, SIAM J. Control Optim. (to be published).

[37] D. Stefanatos and Jr.-Sh. Li, e-print arXiv:1103.1665v1.

[38] H. R. Lewis and P. G. Leach, J. Math. Phys. 23, 2371 (1982).

[39] A. K. Dhara and S. W. Lawande, J. Phys. A 17, 2324 (1984).

[40] L. S. Pontryagin et al., The Mathematical Theory of Optimal Processes (Interscience, New York, 1962).

[41] U. Boscain and P. Mason, J. Math. Phys. 47, 062101 (2006).

[42] R.-B. Wu, J. Dominy, T.-S. Ho, and H. Rabitz, e-print arXiv:0907.2354.

[43] M. Lapert, Y. Zhang, M. Braun, S. J. Glaser, and D. Sugny, Phys. Rev. Lett. 104, 083001 (2010).

[44] R. Grimm, M. Weidenmüller, and Y. B. Ovchinnikov, Adv. At. Mol. Opt. Phys. 42, 95 (2000).

[45] J.-S. Li, J. Ruths, and D. Stefanatos, J. Chem. Phys. 131, 164110 (2009).

[46] J.-S. Li, J. Ruths, T.-Y. Yu, H. Arthanari, and G. Wagner, Proc. Natl. Acad. Sci. 108, 1879 (2011). 